\title{
The Synthetic Effects of Iron with Sulfur and Fluorine on Photoabsorption and Photocatalytic Performance in Codoped $\mathrm{TiO}_{2}$
}

\author{
Xiaohua Li, Jibao Lu, Ying Dai, Meng Guo, and Baibiao Huang \\ School of Physics, State Key Laboratory of Crystal Materials, Shandong University, Jinan 250100, China \\ Correspondence should be addressed to Ying Dai, daiy60@sdu.edu.cn
}

Received 10 August 2011; Accepted 8 September 2011

Academic Editor: Jiaguo Yu

Copyright () 2012 Xiaohua Li et al. This is an open access article distributed under the Creative Commons Attribution License, which permits unrestricted use, distribution, and reproduction in any medium, provided the original work is properly cited.

\begin{abstract}
The structural and electronic properties of iron-fluorine (Fe-F) and iron-sulfur (Fe-S) codoped anatase $\mathrm{TiO}_{2}$ are investigated by first-principles based on density functional theory. Our results show that the formation energy of codoped system is lower than that of single-element doping, which indicates the synergic effect of codoping on the stability of the structure. Codopants introduced impurity gap states resulting in the electron transition energy reduction and thus the visible light absorption observed in the samples. It is concluded that Fe-S should be a better codoping pair because Fe-S codoping introduces extended impurity states resulting in stronger visible light absorption than that of $\mathrm{Fe}-\mathrm{F}$ codoped compounds. This work gives understanding to the recent experiment and provides the evidence of choosing the more effective co-dopants in $\mathrm{TiO}_{2}$.
\end{abstract}

\section{Introduction}

Titanium dioxide has been extensively studied as a promising photocatalyst due to its cheap, stable, and nontoxic characteristics. However, the use of $\mathrm{TiO}_{2}$ is limited by its wide band gap $(\sim 3.0 \mathrm{eV})$ which absorbs only ultraviolet light and accounts for just $5 \%$ of solar energy. Thus, modification of the electronic structure of $\mathrm{TiO}_{2}$ to enable the visible light absorption is of great importance [1-3]. An efficient way is doping pure $\mathrm{TiO}_{2}$ with metal [4-7] or nonmetal elements [8-12]. Metal elements diffused in the titanium lattice greatly enhance the visible light absorption because the impurity states introduced by the dopants lead to the visible light response of $\mathrm{TiO}_{2}$. However, localized states appearing in the band gap of the host semiconductor often result in the recombination of photogenerated carriers and consequently result in lower photocatalytic activity. A possible way to improve the photocatalytic performance of doped $\mathrm{TiO}_{2}$ is to explore the cooperative effect by introducing more than one species of foreign elements to the host [13-16]. Appropriate choice of the codoping pair is the key factor. Recent experiment has reported that iron-sulfur (Fe-S) codoped
$\mathrm{TiO}_{2}$ exhibits quite high photoactivity under visible light illumination and is stable for long-term applications [17]. It is proposed that the doped $\mathrm{Fe}^{3+}$ ions can act as electron acceptor and efficiently prevent electron-hole recombination. In addition, Liu et al. [18] found that iron-fluorine (FeF) codoped $\mathrm{TiO}_{2}$ displays excellent photocatalytic activity under visible light irradiation. In the experiment, they speculated that the as-prepared $\mathrm{TiO}_{2}$ samples match the anatase type, with no trace of rutile or brookite impurity being observed, and they also demonstrated that F could substitute for $\mathrm{O}$ and $\mathrm{Fe}$ could substitute for $\mathrm{Ti}$ in $\mathrm{Fe}-\mathrm{F}$ codoped anatase $\mathrm{TiO}_{2}$. Fluorine atom has one more valence electron than oxygen, and sulfur has the same outer-shell electron as oxygen atom; therefore, it is interesting to explore the origin of the high photocatalytic activity under visible light concerning these two kinds of codoped anatase $\mathrm{TiO}_{2}$.

We should have comprehensive knowledge of singleelement-doped $\mathrm{TiO}_{2}$ before exploiting the cooperative effects of the codoped systems. For Fe-doped $\mathrm{TiO}_{2}$, a number of studies have been reported experimentally and theoretically. Liu et al. [19] demonstrated that Fe-doped $\mathrm{TiO}_{2}$ shows visible light responses and diminished recombination rates 
of the photoexcited carriers. Fe at the 0.5 at.\% level can significantly improve the photoactivity of $\mathrm{TiO}_{2}$ for both oxidation and reduction reactions [20]. As for the F-doped $\mathrm{TiO}_{2}$, recent experimental studies suggested that F-doping neither causes any change in the adsorption edge nor affects the optical absorption of $\mathrm{TiO}_{2}$ but is beneficial to the crystalline anatase phase [21-27]. Umebayashi et al. [28] suggested that $\mathrm{S}$-doping causes the absorption edge of $\mathrm{TiO}_{2}$ to be shifted into lower-energy region. The mixing of the $S 3 p$ states with VB increases the width of the VB itself and results in a decrease in the band gap due to S-doping [29].

In this work, we examine the microscopic electronic structures of $\mathrm{Fe}-\mathrm{S}$ and $\mathrm{Fe}-\mathrm{F}$ codoped anatase $\mathrm{TiO}_{2}$ to explore the synthetic effects of the dopants by means of the firstprinciples density function theory (DFT) calculations. The defect formation energies are calculated to determine which configuration may be realized more easily in experiment. The codoping synergistic effect is specifically elucidated, and the corresponding related properties of Fe-S and Fe-F are compared to identify a better codoping pair. To obtain detailed insight, $\mathrm{Fe}, \mathrm{F}$, and $\mathrm{S}$ monodoped anatase $\mathrm{TiO}_{2}$ structures are also studied systematically. Our theoretical calculations may provide a comprehensive explanation for experimentally observed visible-light photocatalytic activity in the metal and nonmetal codoped $\mathrm{TiO}_{2}$ and may offer some helpful theoretical information for exploiting new effective photocatalysts.

\section{Computational Method}

We carry out the spin-polarized density functional calculations of Fe-S codoped, Fe-F codoped, and single-elementdoped anatase $\mathrm{TiO}_{2}$ using the Vienna ab initio simulation package (VASP) $[30,31]$. The Perdew-Wang 91 of generalized gradient approximation (GGA) is implemented to describe the exchange correlation function. The projectoraugmented wave (PAW) potential is used to represent the electron-ion interaction. The crystal lattice parameters are taken from previous calculations ( $a=3.776, c=9.486$ ), which are in agreement with the experimental values $(a=$ $3.785, c=9.514)$ [32]. We use a $2 \times 2 \times 1$ supercell containing $32 \mathrm{O}$ atoms and $16 \mathrm{Ti}$ atoms to model the bulk anatase $\mathrm{TiO}_{2}$. In the codoped calculation models, one oxygen atom is replaced by a $\mathrm{S}$ (or F) atom and titanium atom by iron. In the mono-doped $\mathrm{TiO}_{2}$, we have just one $\mathrm{O}$ atom substituted by $\mathrm{S}$ (or F). The Monkhorst-Pack k-point is set as $4 \times 4 \times 4$ in the Brillouin zone of the supercell, and we choose the plane-wave cutoff energy of $400 \mathrm{eV}$. All the atoms are fully optimized until the force on each atom is less than $0.1 \mathrm{meV}$.

\section{Results and Discussion}

3.1. Optimized Structure and Stability of Doped $\mathrm{TiO}_{2}$. To investigate the relative stability of Fe-F and Fe-S codoped $\mathrm{TiO}_{2}$, we calculate the defect formation energies. For comparison, the energies of $\mathrm{F}, \mathrm{Fe}$, and $\mathrm{S}$ monodoped $\mathrm{TiO}_{2}$ are also studied. The formation energies $E_{f}$ are calculated according to the following formulas:

$$
\begin{gathered}
E_{f}=E_{\mathrm{Fe}-\text { doped }}-E_{\mathrm{pure}}-\mu_{\mathrm{Fe}}+\mu_{\mathrm{Ti}}, \\
E_{f}=E_{\mathrm{F}(\mathrm{S}) \text {-doped }}-E_{\mathrm{pure}}-\mu_{\mathrm{F}(\mathrm{S})}+\mu_{\mathrm{O}}, \\
E_{f}=E_{\mathrm{Fe} / \mathrm{F}(\mathrm{S}) \text {-codoped }}-E_{\mathrm{pure}}-\mu_{\mathrm{F}(\mathrm{S})}-\mu_{\mathrm{Fe}}+\mu_{\mathrm{O}}+\mu_{\mathrm{Ti}},
\end{gathered}
$$

in which $E_{\mathrm{Fe}-\text { doped }}, E_{\mathrm{F}(\mathrm{S}) \text {-doped }}$, and $E_{\mathrm{Fe} / \mathrm{F}(\mathrm{S}) \text {-codoped }}$ are total energies of $\mathrm{Fe}, \mathrm{F}$ (or $\mathrm{S}$ ) monodoped and $\mathrm{Fe}-\mathrm{F}$ (or $\mathrm{Fe}-\mathrm{S}$ ) codoped $\mathrm{TiO}_{2}$, respectively. $E_{\text {pure }}$ is the total energy of $\mathrm{TiO}_{2}$ without dopants. $\mu_{\mathrm{Fe}}, \mu_{\mathrm{Ti}}, \mu_{\mathrm{F}(\mathrm{S})}$, and $\mu_{\mathrm{O}}$ are the chemical potential of $\mathrm{Fe}, \mathrm{Ti}, \mathrm{F}$ (or $\mathrm{S}$ ), and $\mathrm{O}$, respectively. It is commonly known that if the doped systems have smaller formation energies, it means that they are in a relatively stable phase. It should be also mentioned that formation energy is in connection with crystal growth circumstance. We simulate the corresponding Ti-rich and O-rich conditions in our theoretical calculations. Under Ti-rich condition, $\mu_{\mathrm{Ti}}$ is gotten from bulk $\mathrm{Ti}$ and $\mu_{\mathrm{O}}$ is calculated according to the following formula:

$$
2 \mu_{\mathrm{O}}+\mu_{\mathrm{Ti}}=\mu_{\mathrm{TiO}_{2}} .
$$

Here $\mu_{\mathrm{TiO}_{2}}$ is energy of one formula unit of $\mathrm{TiO}_{2}$. Under Orich, $\mu_{\mathrm{O}}$ can be obtained from the ground-state energy of the $\mathrm{O}_{2}$ molecule $\left(\mu_{\mathrm{O}}=1 / 2 \mu\left(\mathrm{O}_{2}\right)\right)$, while $\mu_{\mathrm{Ti}}$ is fixed by condition (2).

In codoped $\mathrm{TiO}_{2}$ crystal lattice, dopants may substitute any host atoms however, not all of the configurations are stable. With the aim to find out the most stable configuration, different substitution sites according to the different distances between the two dopants are tested. Formation energy calculations imply that the structure with one $\mathrm{Fe}$ substituting for a Ti atom and simultaneously with one $\mathrm{F}$ (or S) at the first nearest neighboring (denoted as $1 \mathrm{NN}$ ) $\mathrm{O}$ atom site is the most energetically favorable. We find that, in general, when the distances between the two dopants increase, the formation energies increase. This is probably because that the attraction forces between the anion and cation ions decrease when the two elements locate far from each other. Thus, it can be concluded that $1 \mathrm{NN}$ substitution is the most viable configuration in experimental process and then is chosen as our computational model hereafter (Figure 1).

The calculated formation energies of different-elementdoped $\mathrm{TiO}_{2}$ are summarized in Table 1 , which suggests that (1) Fe mono-doped, Fe-F and Fe-S codoped $\mathrm{TiO}_{2}$ are both energetically favorable under O-rich condition, but unfavorable under Ti-richcondition, (2) the formation of $\mathrm{F}$ mono-doped $\mathrm{TiO}_{2}$ is thermodynamically favorable under both Ti-rich and O-rich conditions, but $\mathrm{S}$ monodoped $\mathrm{TiO}_{2}$ is more stable under Ti-rich condition, which agrees with the former calculations [11], (3) the formation energy of Fe$\mathrm{F}$ codoped $\mathrm{TiO}_{2}$ is much lower than both $\mathrm{Fe}$ and $\mathrm{F}$ monodoped $\mathrm{TiO}_{2}$. For Fe-S codoping under O-rich condition, the formation energy is smaller than $\mathrm{S}$ mono-doped but larger than $\mathrm{Fe}$ mono-doped $\mathrm{TiO}_{2}$. The lower formation energies of codoping systems indicate cooperative effects 


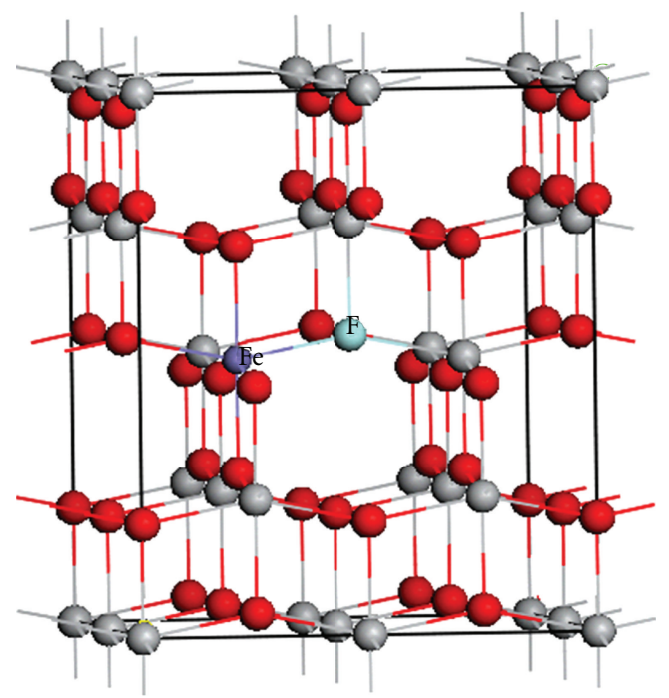

Figure 1: Structure of 48-atom anatase $\mathrm{TiO}_{2}$ codoped with $\mathrm{F}$ and $\mathrm{Fe}$. The gray and red spheres represent $\mathrm{Ti}$ and $\mathrm{O}$ atoms, respectively.

TABLE 1: Formation energies $E_{f}(\mathrm{eV})$ of single-element-doped and codoped $\mathrm{TiO}_{2}$ in different conditions.

\begin{tabular}{lcc}
\hline & \multicolumn{2}{c}{$E_{f}(\mathrm{eV})$} \\
\hline $\mathrm{Fe}-\mathrm{TiO}_{2}$ & 5.51 & O-rich \\
$\mathrm{F}^{-\mathrm{TiO}_{2}}$ & -4.97 & -4.80 \\
$\mathrm{~S}-\mathrm{TiO}_{2}$ & -2.31 & 0.18 \\
$\mathrm{~F}-\mathrm{Fe}-\mathrm{TiO}_{2}$ & -0.19 & 2.85 \\
$\mathrm{Fe}-\mathrm{S}-\mathrm{TiO}_{2}$ & 4.35 & -5.35 \\
\hline
\end{tabular}

of the different dopants and make the high photocatalytic semiconductor with higher dopants concentration more viable in experiment. Thus, we can conclude that codoped $\mathrm{TiO}_{2}$ samples have lower formation energies than monoelement-doped systems, which is due to the charge balance by incorporating both anion and cation ions. This effect is more obvious in $\mathrm{Fe}-\mathrm{F}$ codoped $\mathrm{TiO}_{2}$ since the charge neutrality is well maintained relative to the undoped system, while the charge neutrality is not maintained so well for the Fe-S codoped $\mathrm{TiO}_{2}$.

We further investigate the optimized structures of different-element-doped $\mathrm{TiO}_{2}$. For Fe mono-doped $\mathrm{TiO}_{2}$, the optimized $\mathrm{O}-\mathrm{Fe}$ bond lengths $(1.873 \AA$ and $1.891 \AA)$ are shorter than O-Ti bond lengths in pure $\mathrm{TiO}_{2}(1.930 \AA$ and $1.973 \AA$ ). This is because that bond length is determined mainly by radius and electronegativity of bonded atoms. The electronegativity of iron (1.83) is stronger than that of titanium (1.54), and the ionic radius of iron $(0.64 \AA)$ is smaller than that of titanium $(0.68 \AA)$. In $\mathrm{F}$ mono-doped $\mathrm{TiO}_{2}$, the distance between $\mathrm{F}$ and $\mathrm{Ti}(2.000 \AA)$ is longer than O-Ti bond length (1.930 $\AA$ ). This can be ascribed to the fact that electronegativity of $\mathrm{F}$ (3.98) is stronger than that of $\mathrm{O}$ (3.44) while the radius of $\mathrm{F}^{-}$is a little bigger than that of $\mathrm{O}^{2-}$ (1.32). In S-doped $\mathrm{TiO}_{2}$, the S-Ti bond lengths are 2.147 and $2.354 \AA$, which are much longer than the O-Ti bond lengths in pure $\mathrm{TiO}_{2}$ due to the bigger atom radius of $\mathrm{S}$. As for F-Fe codoped $\mathrm{TiO}_{2}$, the optimized F-Fe bond length is $1.966 \AA, 1.9 \%$ of distortion compared with that of the pure $\mathrm{TiO}_{2}$, which is smaller than the distortions of both F-Ti and $\mathrm{Fe}-\mathrm{O}$ bond length $(3.6 \%$ and $2.8 \%$, resp.). $\mathrm{S}$-Fe bond length $(1.979 \AA)$ in Fe-S codoped $\mathrm{TiO}_{2}$ is longer than that of F-Fe $(1.966 \AA)$, which is due to the bigger atom radius of $S$ than that of $F$. The distortion of optimized $S$ Fe band length $(2.5 \%)$ is smaller than that of both $\mathrm{S}-\mathrm{Ti}$ and $\mathrm{Fe}-\mathrm{O}$ bond length $(11.2 \%$ and $2.8 \%$, resp.) in $\mathrm{S}$ and $\mathrm{Fe}$ mono-doped $\mathrm{TiO}_{2}$. The smaller distortion of codoped $\mathrm{TiO}_{2}$ is in connection with the smaller formation energy, and this should be ascribed to the synergetic effect of codoping.

3.2. Electronic Structures. To clarify how the dopants modify the electronic structure of $\mathrm{TiO}_{2}$, we calculate the total density of states (DOS) and partial density of states (PDOS) of bulk $\mathrm{TiO}_{2}$ and doped $\mathrm{TiO}_{2}$ shown in Figure 2. For pure $\mathrm{TiO}_{2}$ (Figure 2(a)), the calculated band gap is $1.90 \mathrm{eV}$, which is consistent with the previous theoretical studies [33]. Although the theoretical band gap is smaller than the experimental value $(3.2 \mathrm{eV})$ due to the well-known shortcoming of GGA, it is reasonable to analyze the relative variations of the electronic structure without considering the exact band gap value. We can see from Figure $2\left(\mathrm{a}^{\prime}\right)$ that the top of the valence band (VBM) of pure $\mathrm{TiO}_{2}$ consists mainly of $\mathrm{O} 2 p$ states, while the bottom of the conduction band $(\mathrm{CBM})$ is dominated by $\mathrm{Ti} 3 d$ states.

After substitution of fluorine for oxygen atom (Figure 2(b)), the Fermi level is pinned at the bottom of the conduction band which shows a donor character because of one more electron of $\mathrm{F}$ than that of host O. The PDOS (Figure $2\left(b^{\prime}\right)$ ) shows that most of F $2 p$ states are delocalized in the lower-energy range of $\mathrm{VB}$ and do not contribute to the band edge and may not lead to the absorption of visible light. Figure 2(c) shows that incorporation of iron into the lattice results in localized gap states. The Fermi level is pinned at the down-spin orbit of the gap states, which shows half-metallic character. Further projected density of states (PDOS) as shown in Figure $2\left(\mathrm{c}^{\prime}\right)$ predicates that the minority- and majority-spin states within the gap are mainly attributed to $\mathrm{Fe} 3 d$. The impurity states induced above the VBM, below the CBM, and in the forbidden gap are beneficial to the visible light absorption. Figure 2(d) is the DOS of S-doped $\mathrm{TiO}_{2}$, and the VBM has a little shift. From the calculated PDOS of Figure $2\left(\mathrm{~d}^{\prime}\right)$, the valence band is composed mainly of $\mathrm{O} 2 p$, and the conduction band is mainly $\mathrm{Ti} 3 d$. The localized states are generated by $\mathrm{S} 3 p$ about $0.7 \mathrm{eV}$ above the VBM relative to the undoped one. The excitation from these occupied S $3 p$ states to conduction band might lead to a decrease of the photon excitation energy and induce more significant red shift of absorption, which is consistent with experimental absorption spectra measurements. However, the localized gap states in the middle of the forbidden band in Fe-doped $\mathrm{TiO}_{2}$ provide recombination center of photogenerated electron-hole pair, which is detrimental to the photocatalytic activity. The improvement of photocatalytic activity originating from visible light absorption is weakened by the increase of 

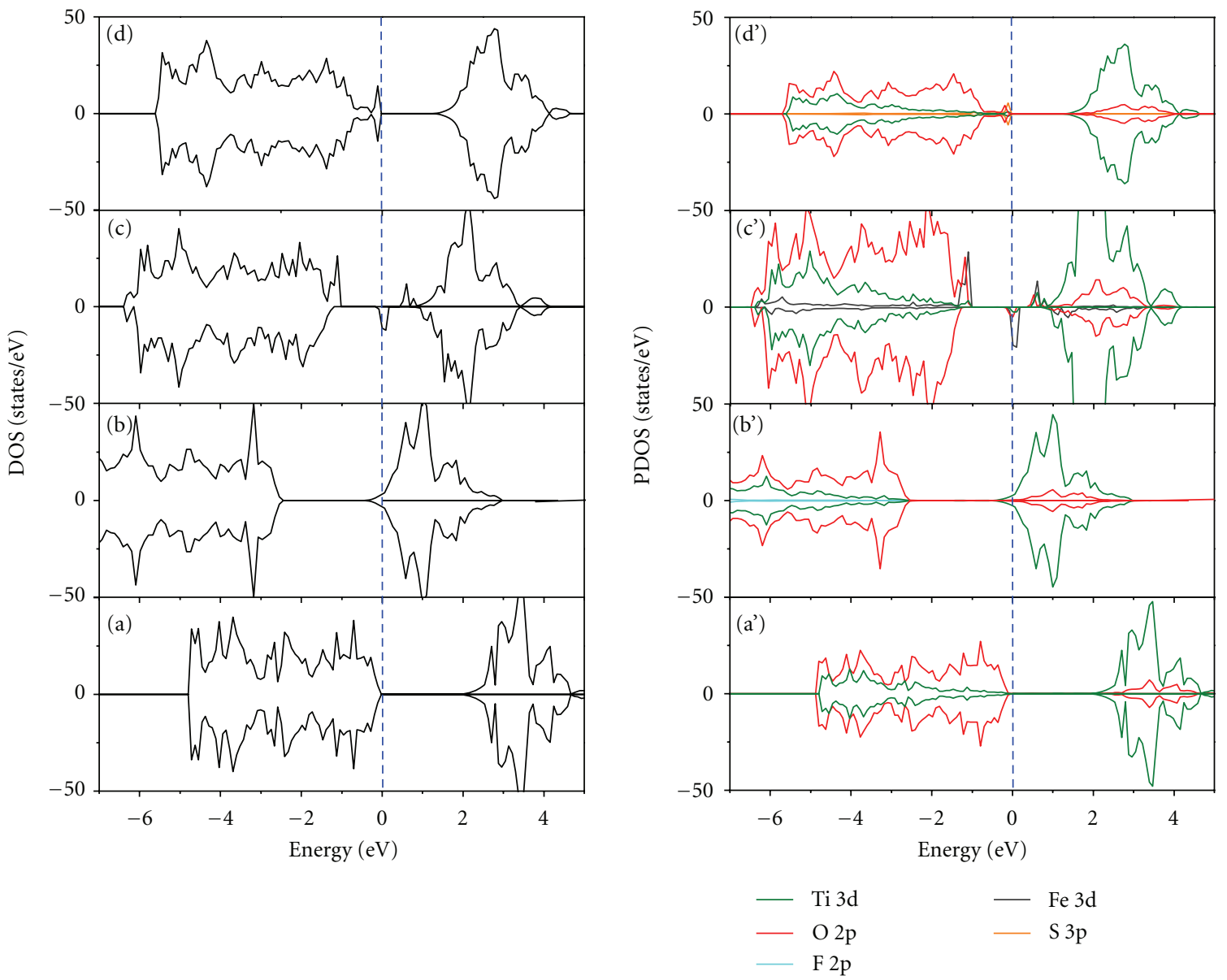

Figure 2: Total density of states (DOS) of (a) undoped anatase $\mathrm{TiO}_{2}$, (b) F-doped $\mathrm{TiO}_{2}$, (c) Fe-doped $\mathrm{TiO}_{2}$, and (d) S-doped TiO 2 and projected density of states (PDOS) of $\left(\mathrm{a}^{\prime}\right)$ pure $\mathrm{TiO}_{2}$, ( $\left.\mathrm{b}^{\prime}\right)$ F-doped $\mathrm{TiO}_{2}$, (c' $\left.\mathrm{c}^{\prime}\right)$ Fe-doped $\mathrm{TiO}_{2}$, and $\left(\mathrm{d}^{\prime}\right) \mathrm{S}$ doped $\mathrm{TiO}_{2}$. The dashed line represents the Fermi energy level.

recombination of the carriers and eventually limits the great enhancement of photocatalytic activity.

For Fe-F codoped $\mathrm{TiO}_{2}$ (Figure 3(a)), localized impurity states are introduced between VBM and CBM. The PDOS in Figure $3\left(a^{\prime}\right)$ demonstrates that three gap states mainly stem from $\mathrm{Fe} 3 d$ orbital. The energies needed for electrons excitation from two occupied up-spin states to the CBM are 1.6 and $0.9 \mathrm{eV}$, respectively, while the energy from down-spin state to $\mathrm{CBM}$ is $0.5 \mathrm{eV}$. Hence, the electron transition from these impurity energy levels to the conduction band would lead to an obvious reduction of absorption energy. Our results give a good explanation for the experimentally observed red shift of absorption edge of the Fe-F codoped anatase $\mathrm{TiO}_{2}$ [18]. However, as the Fe mono-doped structure, localized states introduced in the middle of the forbidden band can lead to the recombination of electro-hole pair and thus do harm to the photocatalytic activity. Therefore, even though codoping with $\mathrm{Fe}$ and $\mathrm{F}$ may promote the incorporation of dopants into the $\mathrm{TiO}_{2}$ host lattice, it will not have pronounced enhancement in the photocatalytic activity compared with Fe-doped $\mathrm{TiO}_{2}$.

When $\mathrm{Fe}$ and $\mathrm{S}$ are introduced into $\mathrm{TiO}_{2}$ simultaneously, more obvious spin polarization can be observed in the band edge compared with mono-doped $\mathrm{TiO}_{2}$ and localized gap states appear about $0.2 \mathrm{eV}$ above the valence band with width of $0.4 \mathrm{eV}$ and $0.2 \mathrm{eV}$ below the conduction band with width of $0.6 \mathrm{eV}$ (Figure 3(b)). The bandwidth decreases to $1.7 \mathrm{eV}$, $0.2 \mathrm{eV}$ smaller than that of pure $\mathrm{TiO}_{2}$, and the Fermi level is pinned in the gap states located below the conduction band due to the slight break of the charge neutrality by Fe-S codoping. Electron excitation from VBM to the gap states and CBM could lead to the visible light absorption as observed in experiment [17]. The PDOS shown in Figure $3\left(b^{\prime}\right)$ indicates that the gap states above the valence band are the mixing of Fe $3 d, \mathrm{O} 2 p$, and $\mathrm{S} 3 p$ orbitals while the states below the CBM are mainly hybridization of Fe $3 d$ and $\mathrm{O} 2 p$. The formation energy reduction is found in both Fe-F and $\mathrm{Fe}-\mathrm{S}$ codoped $\mathrm{TiO}_{2}$ which could enhance the solubility of dopants in the host lattice. Furthermore, the codoping of $\mathrm{Fe}$ and $S$ produces extended states near the band edge and does not induce localized states in the center of the gap that often act as recombination centers. This electronic structure could enhance the visible light absorption and reduce the recombination of photogenerated electron-hole pairs. Moreover, the impurity states near the band edge are more extended than those of mono-doped and Fe-F-doped structures, which 

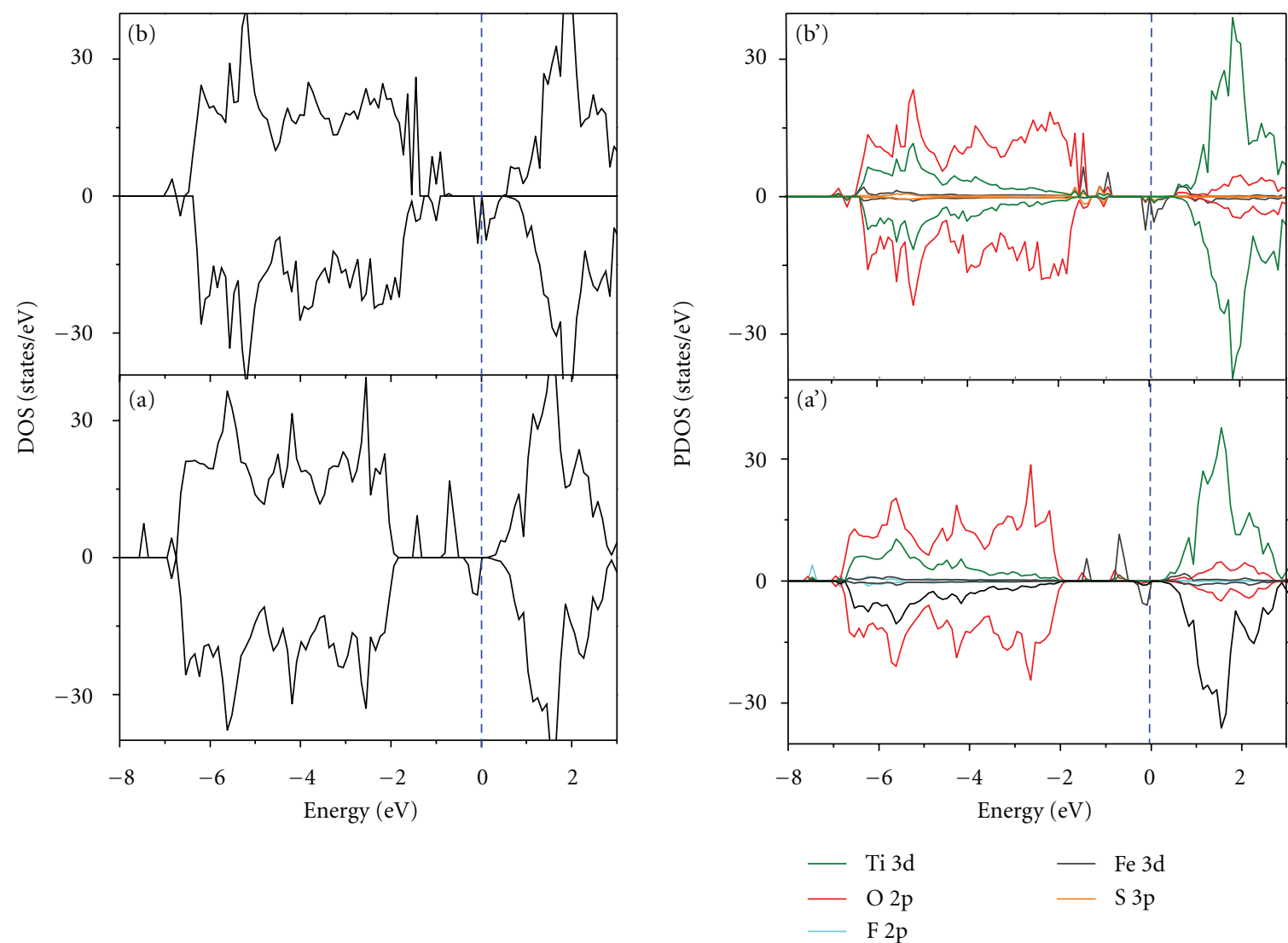

FIgure 3: Total density of states (DOS) of (a) Fe-F codoped $\mathrm{TiO}_{2}$ and (b) Fe-S codoped $\mathrm{TiO}_{2}$ and projected density of states (PDOS) of (a') Fe-F codoped $\mathrm{TiO}_{2}$ and $\left(b^{\prime}\right)$ Fe-S codoped $\mathrm{TiO}_{2}$. The dashed line represents the Fermi energy level.

means that electron excitation between the valence band (conduction band) and impurity states can be more intense, and consequently the intensity of visible light absorption can be stronger. It should be mentioned that the mobility of the photo-carriers in the impurity states is lower than that in the valence band of pure $\mathrm{TiO}_{2}$; however, the impurity states near the band edge can also act as electron/hole traps, which reduces the recombination of photocarriers. Additionally, since the oxidation (reduction) power of photogenerated holes (electrons) in the gap states is reduced relative to that in the VB (CB) of pure $\mathrm{TiO}_{2}$, one should accommodate a balance between the oxidation (reduction) power and visible light absorption of the photocatalysts.

Based on these analyses, we conclude that the Fe-S codoped $\mathrm{TiO}_{2}$ could possess the best photocatalytic activity under visible light irradiation among the $\mathrm{Fe}$ mono-doped and $\mathrm{Fe}-\mathrm{F}, \mathrm{Fe}-\mathrm{S}$ codoped structures.

\section{Conclusions}

We have examined the crystal structures and electronic and optical properties of Fe-F and Fe-S codoped anatase $\mathrm{TiO}_{2}$ based on DFT calculations. For comparison, Fe, S, and F single -doped $\mathrm{TiO}_{2}$ are also studied. Formation energy of the codoped system is much lower than that of the monoelement doping indicating the synergic effects of codopants on the stability of the doped structure. The calculated results indicate that the codoped atoms introduce impurity energy levels in the band gap mainly composed of Fe $3 d$ states. Due to the less energy needed for an electron transition from the impurity energy levels to the conduction band bottom, codoped anatase $\mathrm{TiO}_{2}$ may show higher photocatalytic activity than the mono-doped one under visible light, which may account for the experimentally observed phenomenon. However, Fe-F codoping introduced localized gap states which may result in visible light absorption but decline the photocatalytic activity. Compared with Fe-F codoping, Fe-S codoped $\mathrm{TiO}_{2}$ produces gap states near the band edge that are extended and may greatly enhance the visible light absorption and reduce the carrier recombination. Consequently, the photocatalytic performance under visible light of Fe-S codoped $\mathrm{TiO}_{2}$ is better than that of Fe$\mathrm{F}$ codoped one, and Fe-S should be a better codoping pair.

\section{Acknowledgments}

This work is supported by the National Basic Research Program of China (973 program, 2007CB613302), the National Science Foundation of China under Grant nos. 11174180 and 20973102, and the Natural Science Foundation of Shandong Province under Grant no. ZR2011AM009. 


\section{References}

[1] J. Lu, Y. Dai, H. Jin, and B. Huang, "Effective increasing of optical absorption and energy conversion efficiency of anatase $\mathrm{TiO}_{2}$ nanocrystals by hydrogenation," Physical Chemistry Chemical Physics, vol. 13, no. 40, pp. 18063-18068, 2011.

[2] G. Shang, H. Fu, S. Yang, and T. Xu, "Mechanistic study ofvisible-light-induced photodegradation of 4-chlorophenol by $\mathrm{TiO}_{2}-\mathrm{xNx}(0.021<\mathrm{x}<0.049)$ with low nitrogen concentration," International Journal of Photoenergy, vol. 2012, Article ID 759306, 9 pages, 2012.

[3] J. A. Byrne, P. A. Fernandez-Ibañez, P. S. M. Dunlop, D. M. A. Alrousan, and J. W. J. Hamilton, "Photocatalytic enhancement for solar disinfection of water: a review," International Journal of Photoenergy, vol. 2011, Article ID 798051, 12 pages, 2011.

[4] L. Jing, B. Xin, F. Yuan, L. Xue, B. Wang, and H. Fu, "Effects of surface oxygen vacancies on photophysical and photochemical processes of $\mathrm{Zn}$-doped $\mathrm{TiO}_{2}$ nanoparticles and their relationships," Journal of Physical Chemistry B, vol. 110, no. 36, pp. 17860-17865, 2006.

[5] S. Zhu, L. M. Wang, X. T. Zu, and X. Xiang, "Optical and magnetic properties of Ni nanoparticles in rutile formed by $\mathrm{Ni}$ ion implantation," Applied Physics Letters, vol. 88, no. 4, Article ID 043107, pp. 1-3, 2006.

[6] L. F. Fu, N. D. Browning, S. X. Zhang, S. B. Ogale, D. C. Kundaliya, and T. Venkatesan, "Defects in Co-doped and (Co, $\mathrm{Nb}$ )-doped $\mathrm{TiO}_{2}$ ferromagnetic thin films," Journal of Applied Physics, vol. 100, no. 12, Article ID 123910, 2006.

[7] A. di Paola, S. Ikeda, G. Marcì, B. Ohtani, and L. Palmisano, "Transition metal doped $\mathrm{TiO}_{2}$ : physical properties and photocatalytic behaviour," International Journal of Photoenergy, vol. 3, no. 4, pp. 171-176, 2001.

[8] R. Asahi, T. Morikawa, T. Ohwaki, K. Aoki, and Y. Taga, "Visible-light photocatalysis in nitrogen-doped titanium oxides," Science, vol. 293, no. 5528, pp. 269-271, 2001.

[9] C. di Valentin, G. Pacchioni, A. Selloni, S. Livraghi, and E. Giamello, "Characterization of paramagnetic species in $\mathrm{N}$ doped $\mathrm{TiO}_{2}$ powders by EPR spectroscopy and DFT calculations," Journal of Physical Chemistry B, vol. 109, no. 23, pp. 11414-11419, 2005.

[10] T. Umebayashi, T. Yamaki, H. Itoh, and K. Asai, "Band gap narrowing of titanium dioxide by sulfur doping," Applied Physics Letters, vol. 81, no. 3, p. 454, 2002.

[11] K. Yang, Y. Dai, B. Huang, and M. H. Whangbo, "Density functional characterization of the band edges, the band gap states, and the preferred doping sites of halogen-doped $\mathrm{TiO}_{2}$," Chemistry of Materials, vol. 20, no. 20, pp. 6528-6534, 2008.

[12] V. K. Sharma, K. Winkelmann, Y. Krasnova, C. Lee, and M. Sohn, "Heterogeneous photocatalytic reduction of ferrate(VI) in UV-irradiated titania suspensions: role in enhancing destruction of nitrogen-containing pollutants," International Journal of Photoenergy, vol. 5, no. 3, pp. 183-190, 2003.

[13] L. Jia, C. Wu, Y. Li et al., "Enhanced visible-light photocatalytic activity of anatase $\mathrm{TiO}_{2}$ through $\mathrm{N}$ and $\mathrm{S}$ codoping," Applied Physics Letters, vol. 98, no. 21, Article ID 211903, 2011.

[14] M. Xing, Y. Wu, J. Zhang, and F. Chen, "Effect of synergy on the visible light activity of $\mathrm{B}, \mathrm{N}$ and $\mathrm{Fe}$ co-doped $\mathrm{TiO}_{2}$ for the degradation of MO," Nanoscale, vol. 2, no. 7, pp. 1233-1239, 2010 .

[15] W. Wei, Y. Dai, M. Guo, L. Yu, and B. Huang, "Density functional characterization of the electronic structure and optical properties of N-doped, La-doped, and N/La-codoped $\mathrm{SrTiO}_{3}$," Journal of Physical Chemistry C, vol. 113, no. 33, pp. 1504615050, 2009.
[16] W. Wei, Y. Dai, M. Guo et al., "Codoping synergistic effects in $\mathrm{N}$-doped $\mathrm{SrTiO}_{3}$ for higher energy conversion efficiency," Physical Chemistry Chemical Physics, vol. 12, no. 27, pp. 76127619, 2010.

[17] V. M. Menéndez-Flores, D. W. Bahnemann, and T. Ohno, "Visible light photocatalytic activities of S-doped $\mathrm{TiO}_{2}-\mathrm{Fe}^{3+}$ in aqueous and gas phase," Applied Catalysis B, vol. 103, no. 1-2, pp. 99-108, 2011.

[18] S. Liu, X. Sun, J. G. Li et al., "Fluorine- and ironmodified hierarchical anatase microsphere photocatalyst for water cleaning: facile wet chemical synthesis and wavelengthsensitive photocatalytic reactivity," Langmuir, vol. 26, no. 6, pp. 4546-4553, 2010.

[19] Y. Liu, J. H. Wei, R. Xiong, C. X. Pan, and J. Shi, "Enhanced visible light photocatalytic properties of Fe-doped $\mathrm{TiO}_{2}$ nanorod clusters and monodispersed nanoparticles," Applied Surface Science, vol. 257, no. 18, pp. 8121-8126, 2011.

[20] W. Choi, A. Termin, and M. R. Hoffmann, "The role of metal ion dopants in quantum-sized $\mathrm{TiO}_{2}$ : correlation between photoreactivity and charge carrier recombination dynamics," Journal of Physical Chemistry, vol. 98, no. 51, pp. 13669-13679, 1994.

[21] D. Li, H. Haneda, S. Hishita, N. Ohashi, and N. K. Labhsetwar, "Fluorine-doped $\mathrm{TiO}_{2}$ powders prepared by spray pyrolysis and their improved photocatalytic activity for decomposition of gas-phase acetaldehyde," Journal of Fluorine Chemistry, vol. 126, no. 1, pp. 69-77, 2005.

[22] D. Li, H. Haneda, S. Hishita, and N. Ohashi, "Visible-lightdriven N-F-codoped $\mathrm{TiO}_{2}$ photocatalysts. 2. Optical characterization, photocatalysis, and potential application to air purification," Chemistry of Materials, vol. 17, no. 10, pp. 25962602, 2005.

[23] A. Olasz, P. Mignon, F. Deproft, T. Veszprémi, and P. Geerlings, "Effect of the $\pi-\pi$ stacking interaction on the acidity of phenol," Chemical Physics Letters, vol. 407, no. 4-6, pp. 504509, 2005.

[24] J. K. Zhou, L. Lv, J. Yu et al., "Synthesis of self-organized polycrystalline F-doped $\mathrm{TiO}_{2}$ hollow microspheres and their photocatalytic activity under visible light," Journal of Physical Chemistry C, vol. 112, no. 14, pp. 5316-5321, 2008.

[25] D. G. Huang, S. J. Liao, J. M. Liu, Z. Dang, and L. Petrik, "Preparation of visible-light responsive N-F-codoped $\mathrm{TiO}_{2}$ photocatalyst by a sol-gel-solvothermal method," Journal of Photochemistry and Photobiology A, vol. 184, no. 3, pp. 282288, 2006.

[26] D. Huang, S. Liao, S. Quan et al., "Preparation of anatase F doped $\mathrm{TiO}_{2}$ sol and its performance for photodegradation of formaldehyde," Journal of Materials Science, vol. 42, no. 19, pp. 8193-8202, 2007.

[27] J. Tang, H. Quan, and J. Ye, "Photocatalytic properties and photoinduced hydrophilicity of surface-fluorinated $\mathrm{TiO}_{2}$," Chemistry of Materials, vol. 19, no. 1, pp. 116-122, 2007.

[28] T. Umebayashi, T. Yamaki, H. Itoh, and K. Asai, "Band gap narrowing of titanium dioxide by sulfur doping," Applied Physics Letters, vol. 81, no. 3, p. 454, 2002.

[29] T. Umebayashi, T. Yamaki, S. Yamamoto et al., "Sulfur-doping of rutile-titanium dioxide by ion implantation: photocurrent spectroscopy and first-principles band calculation studies," Journal of Applied Physics, vol. 93, no. 9, pp. 5156-5160, 2003.

[30] G. Kresse and J. Furthmüller, "Efficient iterative schemes for ab initio total-energy calculations using a plane-wave basis set," Physical Review B, vol. 54, no. 16, pp. 11169-11186, 1996.

[31] G. Kresse and J. Furthmüller, "Efficiency of ab-initio total energy calculations for metals and semiconductors using a 
plane-wave basis set," Computational Materials Science, vol. 6, no. 1, pp. 15-50, 1996.

[32] J. Zhu, Z. Deng, F. Chen et al., "Hydrothermal doping method for preparation of $\mathrm{Cr}^{3+}-\mathrm{TiO}_{2}$ photocatalysts with concentration gradient distribution of $\mathrm{Cr}^{3+}$," Applied Catalysis B, vol. 62, no. 3-4, pp. 329-335, 2006.

[33] K. Yang, Y. Dai, and B. Huang, "Understanding photocatalytic activity of S- and P-doped $\mathrm{TiO}_{2}$ under visible light from firstprinciples," Journal of Physical Chemistry C, vol. 111, no. 51, pp. 18986-18994, 2007. 


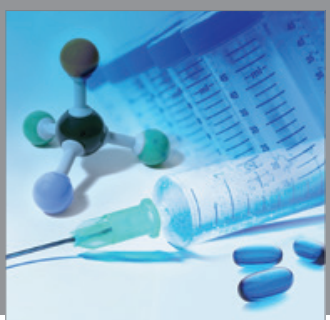

International Journal of

Medicinal Chemistry

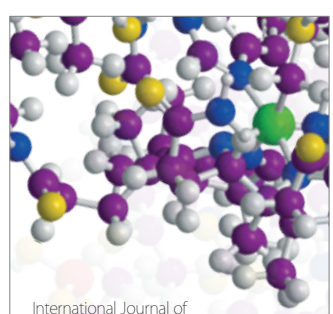

Carbohydrate Chemistry

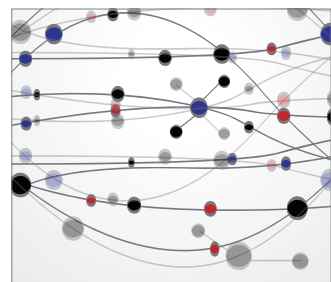

The Scientific World Journal
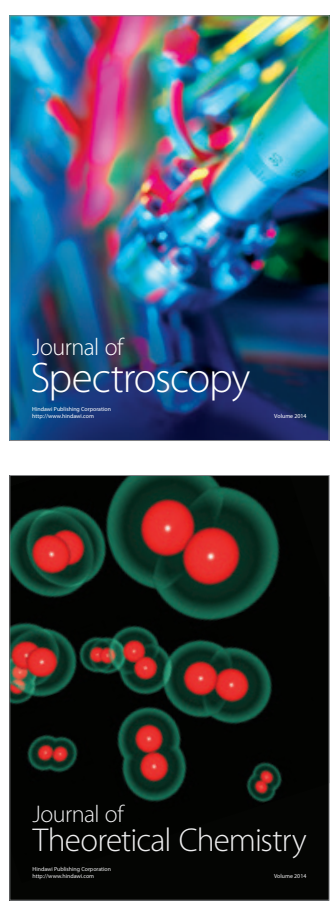
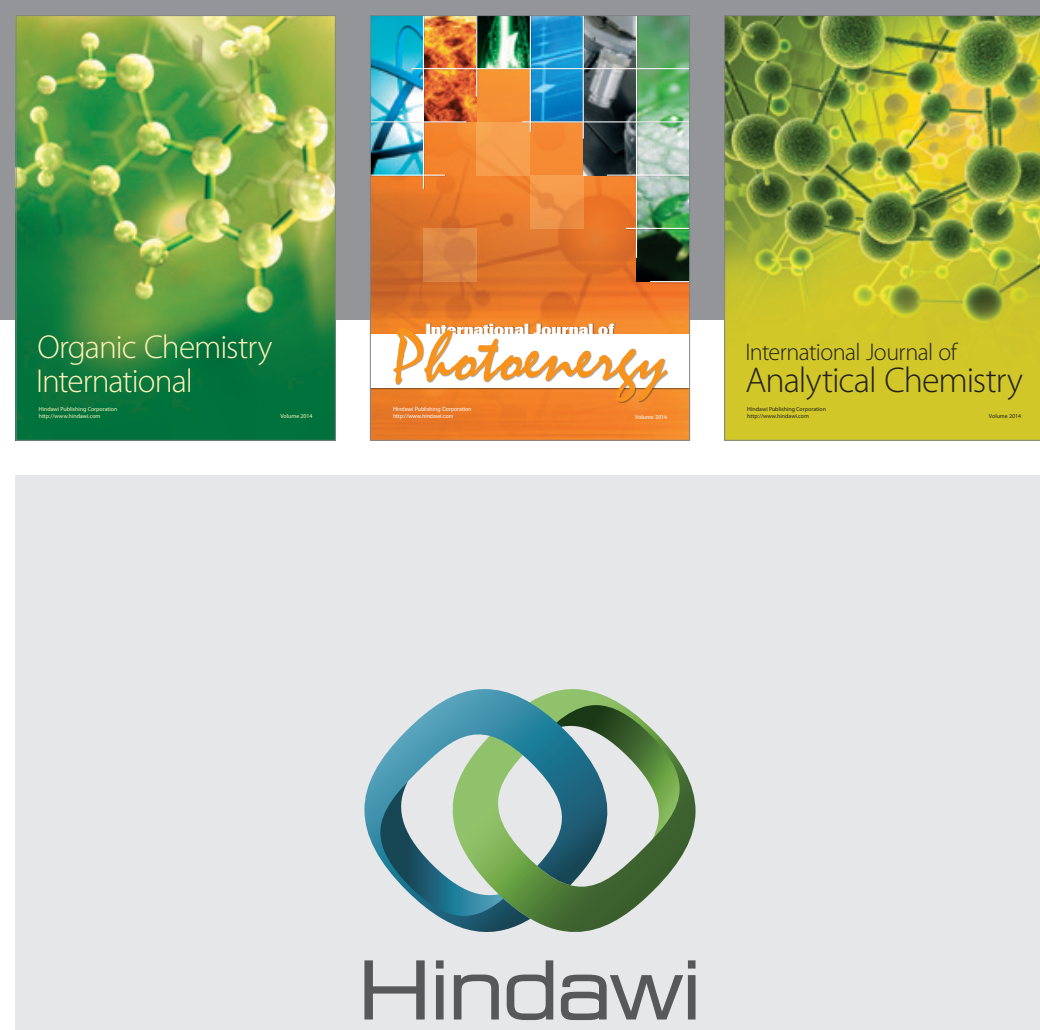

Submit your manuscripts at

http://www.hindawi.com
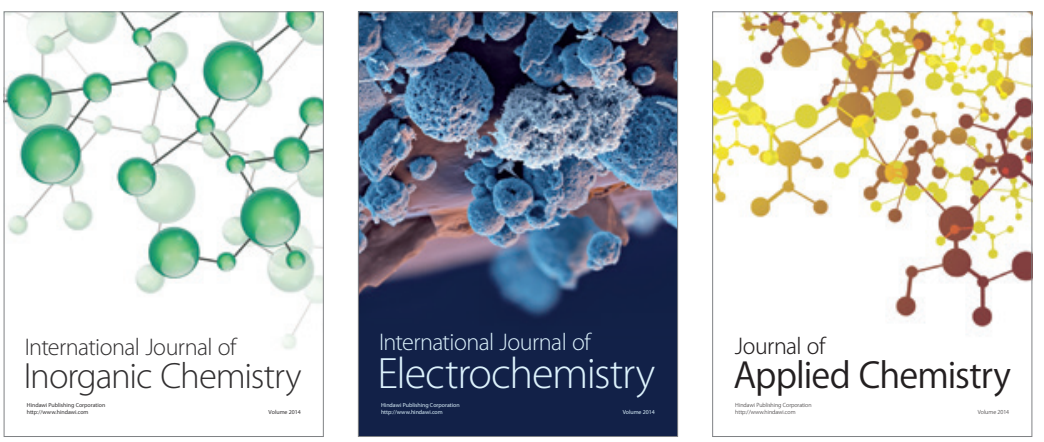

Journal of

Applied Chemistry
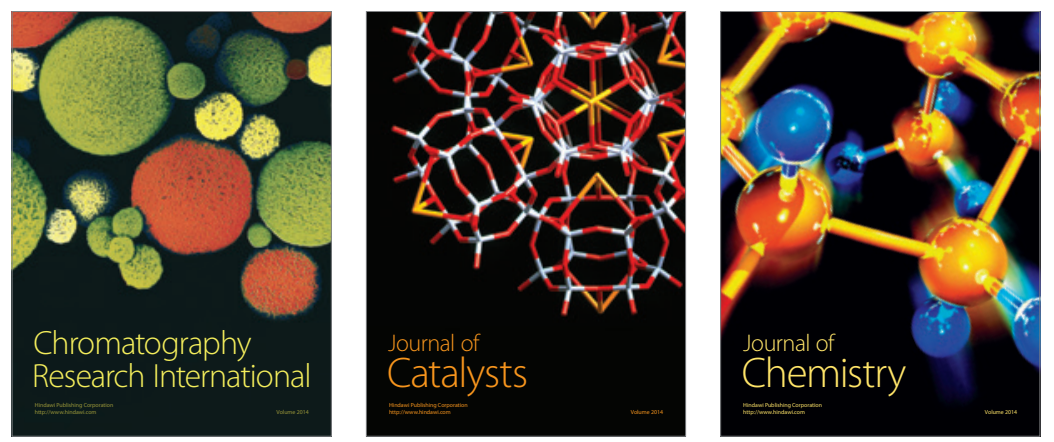
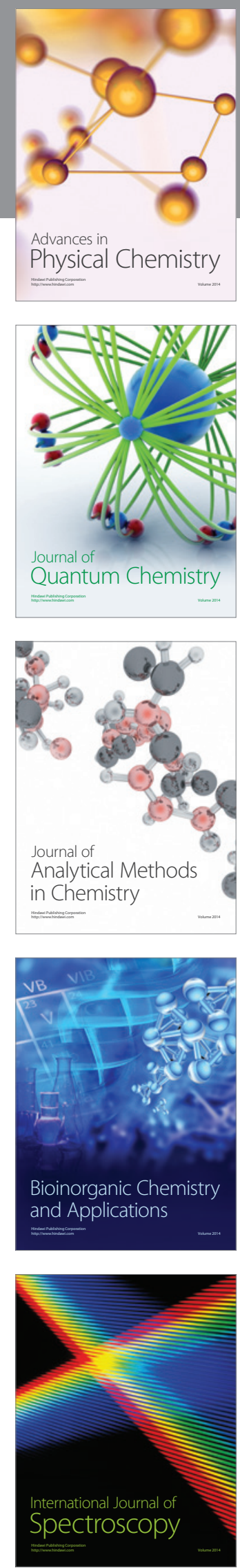